\title{
How a visual surveillance system hypothesizes how you behave
}

\author{
C. MICHELONI, C. PICIARELLI, and G. L. FORESTI \\ University of Udine, Udine, Italy
}

\begin{abstract}
In the last few years, the installation of a large number of cameras has led to a need for increased capabilities in video surveillance systems. It has, indeed, been more and more necessary for human operators to be helped in the understanding of ongoing activities in real environments. Nowadays, the technology and the research in the machine vision and artificial intelligence fields allow one to expect a new generation of completely autonomous systems able to reckon the behaviors of entities such as pedestrians, vehicles, and so forth. Hence, whereas the sensing aspect of these systems has been the issue considered the most so far, research is now focused mainly on more newsworthy problems concerning understanding. In this article, we present a novel method for hypothesizing the evolution of behavior. For such purposes, the system is required to extract useful information by means of low-level techniques for detecting and maintaining track of moving objects. The further estimation of performed trajectories, together with objects classification, enables one to compute the probability distribution of the normal activities (e.g., trajectories). Such a distribution is defined by means of a novel clustering technique. The resulting clusters are used to estimate the evolution of objects' behaviors and to speculate about any intention to act dangerously. The provided solution for hypothesizing behaviors occurring in real environments was tested in the context of an outdoor parking lot.
\end{abstract}

The deployment of a large number of video sensors in such environments as stations, airports, and shopping malls has progressively required the development of automatic surveillance systems able to handle the large quantity of data they are capable of acquiring.

Hence, the development of an autonomous surveillance system now requires facing such problems as efficient image transmission (Mahonen, 1999), powerful image processing, and semantic image storage and retrieval (Enser, 2000). The main issue is that all of these points must be carried out in real time, to meet the requirements of a watchful system.

Moreover, as the electronic market has evolved, with the production of more and more powerful sensors and computers at a cheaper price, the system architectures (Collins, Lipton, Fujiyoshi, \& Kanade, 2001) have become really complex by introducing a combination of heterogeneous sensors. Now, surveillance systems should be thought of as networks of sensors.

The increasing complexity of the architectures has introduced new problems to be solved (i.e., image transmission, flow synchronization, multisensor analysis, image storage, etc.). Thus, the development of an advanced video surveillance system can be supported on three different dimensions: (1) image transmission, (2) image processing/ analysis, and (3) image storage and retrieval. Although all of these aspects have been well considered in the past, the

Correspondence concerning this article should be addressed to C. Piciarelli, Dipartimento di Matematica e Informatica, Università degli Studi di Udine, Via delle Scienze 206, 33100 Udine, Italy (e-mail: piccia@dimi .uniud.it). one that has certainly prompted the biggest effort from the computer vision community has consisted of the processing of the images acquired for understanding purposes. Actually, this is the most important task, since it is the closest to the artificial intelligence concept.

In this field, attention has principally been focused on low-level algorithms. With this term, we mean all those algorithms that allow one to extract useful information for the understanding of activities directly from the raw image data. As a result, information gathered by low-level computations can be exploited by high-level modules to extract events. Hence, event detection yields to event understanding, which is strictly related to the understanding of behaviors.

The advances in low-level processing have delivered a large set of algorithms that, nowadays, are considered robust enough to shift the focus to the high-level techniques, even if algorithms for the detection and tracking of moving objects still need to be polished. They are based mainly on image-differencing techniques (Foresti, Micheloni, Snidaro, Remagnino, \& Ellis, 2005) - thus, comparing the current image with a background model-and are able to segment moving objects (foreground) from the environment (background). Afterward, detected objects are localized by computing their current position inside a map of the monitored environment. Localization is useful information for the next tracking step, in which the same object is tracked through time, discriminating it from other, similar objects in the scene. Tracking yields to a definition of the trajectory performed by each entity. In this work, such trajectories are used to provide hypotheses about the activities occurring within the monitored space. 
Furthermore, since the behavior of an entity is strongly linked to its type, recognition techniques (Foresti \& Gentili, 1999) have been considered that classify objects into predefined categories and, therefore, trigger an opportune analysis of their behaviors.

It is, hence, well understood that behavior analysis depends on low-level analysis modules (i.e., detection, localization, tracking, recognition, etc.). They, indeed, represent the foundation bricks on which to build robust high-level algorithms for event detection and event recognition.

In particular, it is required that those events be detected that are considered of paramount importance for inferring behaviors. In such a context, in the last years, some researchers have provided some interesting solutions. Buxton and Gong (1995) have introduced Bayesian networks for detecting interesting events in a dynamic scene and providing interpretations of traffic situations. Brand, Oliver, and Pentland (1997) have proposed the use of probabilistic models (i.e., hidden Markov models) to capture the uncertainty of moving object properties, and Chleq and Thonnat (1996) have proposed a generic framework for the real-time interpretation of real-world scenes utilized by humans. Detected events can, therefore, be exploited to dynamically depict a scene. Herzog (1992) has described a temporal sequence of events by using an inference method based on time interval logic. The main issue concerns the fact that its aim is to describe human behaviors in environments (e.g., soccer games) when group behavior understanding would be more suitable. Ayers and Shah (1998) have used a similar approach to describe human activities in an office environment.

Although useful, behavior analysis based on event association and temporal correlation is not reliable enough to describe the complex interactions occurring in real environments, such as those we want to analyze. In effect, the definition of simple events represents a substantial drawback, since it is quite impossible to identify all the events of interest.

In this article, we propose a new method for understanding activities occurring inside a complex outdoor environment, such as a parking lot. As is shown in Figure 1, the proposed system ranges from low-level processing up to high-level behavior analysis. Low-level procedures involve object detection, tracking (see the Low-Level Analysis section) and classification (see the Object Classification section). Trajectories are then clustered (see the Trajectory Clustering section) to organize data so that they can be more useful and treatable for high-level processing. Finally, we propose two different approaches for behavioral event detection and classification (see the Behavior Understanding section). The first tack is based on a probabilistic analysis of the acquired data (in particular, trajectories), whereas the second one is based on the explicit modeling of suspicious or dangerous events.

\section{LOW-LEVEL ANALYSIS}

In the proposed system, CCD progressive color cameras are used to acquire image sequences of the monitored

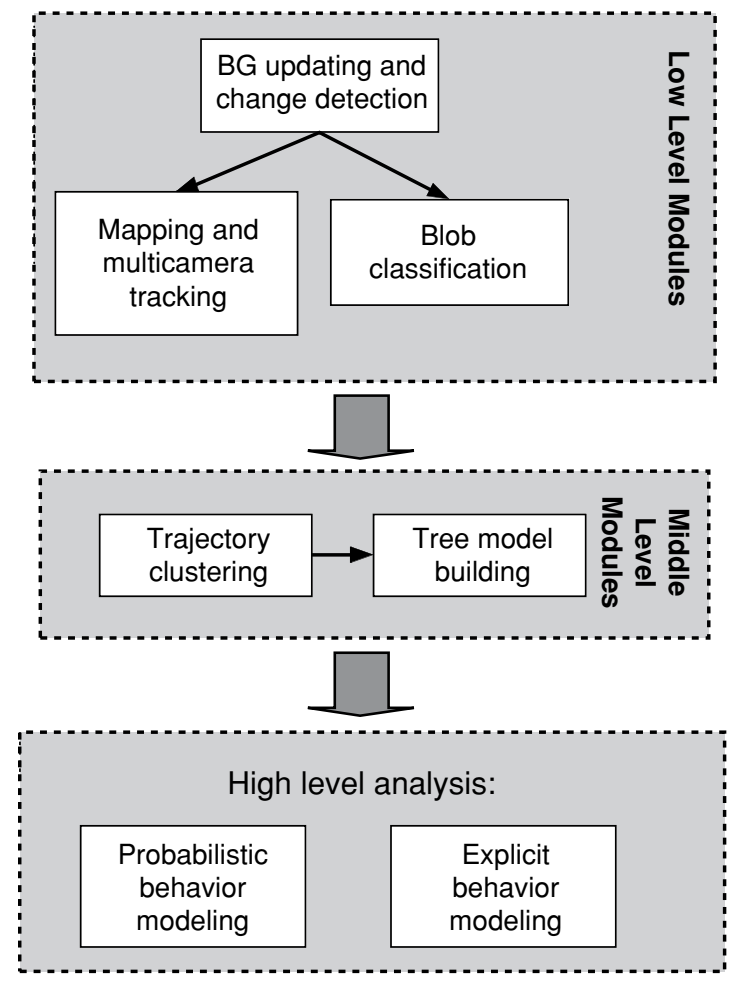

Figure 1. System architecture.

environment. Figure 2A shows a typical scene of a parking lot where multiple moving objects (e.g., pedestrians, vehicles, cycles, etc.) can be found. A change detection (CD) module (Mahonen, 1999) is applied to compare each frame $[I(x, y)]$ of the input image sequence with a background image $[B C K(x, y)]$. An automatic thresholding technique (Rosin, 2002) is applied to find the best threshold value. A background-updating module based on the Kalman filter is used to adapt the pixels of the background image to significant changes in the scene. Such a technique allows one to achieve good results even for significant changes in the background, such as those due to slowly moving shadows or changing lighting conditions. The main drawback of this technique is the presence of ghosts (objects belonging to the background model but not to the real background). They appear when a static object (especially a car) starts moving and last for a certain amount of time before being absorbed by the updating technique. This time is subject to thresholding, in order to have a fast or a slow updating.

The CD procedure generates a binary image $[B(x, y)]$ in which pixels can assume two possible states: a background or a moving object point. Groups of connected moving points represent possible objects (e.g., vehicles, pedestrians, etc.) moving in the scene. These groups of pixels are commonly called blobs. Grayscale or color pixels belonging to the blob area in the input image can also be considered to increase the information about the detected object. Figure 2B shows the blobs extracted from the input image in Figure 2A. 
(A)

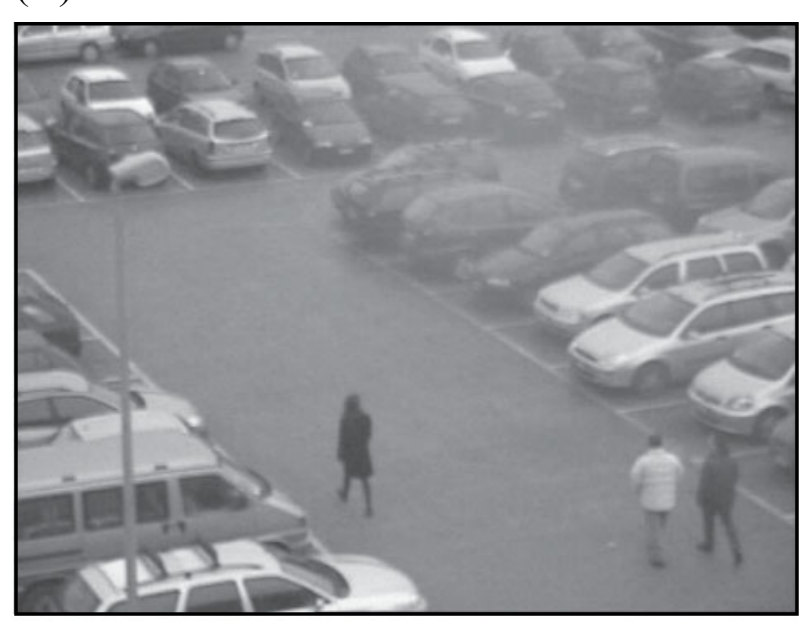

(B)



Figure 2. (A) A parking lot and (B) the extracted blobs.

The image-processing techniques above are applied for each static camera in the system. In addition to the set of static cameras, we also adopted an active camera approach. That is, the camera parameters (pan/tilt position and zoom level) are dynamically tuned to perform the active tracking of an object of interest (the target). By means of these active vision techniques, it is possible to adapt the image acquisition process for security purposes. Moreover, the ability to maintain the gaze on the target, as well as to acquire it at higher resolutions, increases the awareness of the monitored scene.

Such a property turns out to be fundamental to supporting the behavior understanding process, with particular regard to wide areas. Although active cameras can acquire targets at higher resolutions, their field of view (FOV) is narrower than those in the static cameras case. In particular, if they are exploited for face recognition or gesture analysis, their limited FOV allows the tracking of only one object in the scene. It is, therefore, of primary importance to select the target in such a way that its behavior is considered unusual. As was said before, in this work, we adopted a novel method of classifying trajectories in order to estimate the evolution of object behaviors. It is for such purposes that the static cameras network needs to maintain track of all the objects that are considered active inside a monitored scene.

To track all the objects, a first processing occurs locally at each image plane. Hence, for each sensor, the system applies an association algorithm for matching the currently detected blobs with those extracted in the previous frame. Generally, a 2-D top view map of the monitored environment is taken as a common coordinate system, and the position of the objects identified by each camera is projected onto the map. Data obtained from different sensors are, therefore, fused together to yield a better estimate of the object positions. A measurement fusion approach based on a Kalman filter (Gao \& Harris, 2002) has been employed for this purpose. Such a scheme involves the fusion of the target position (according to different sensors) obtained by the coordinate conversion function. This approach can be successfully exploited in all those scenes in which the number of objects allows single-object detection for all the frames and all the cameras, except for short time instants, when occlusions can be handled by means of estimation.

\section{OBJECT CLASSIFICATION}

The function of the object classification module is to classify each detected object according to a predefined set of categories (e.g., cars, pedestrians, and cycles in outdoor environments). In the proposed system, this is obtained by using an adaptive high-order neural tree (AHNT) classifier (Foresti \& Dolso, 2004). The AHNT is a hierarchical multilevel neural network, in which the nodes are organized into a tree topology. Each node of the tree can itself be a high-order perceptron or a first-order perceptron, depending on the local training complexity. Locally, each node receives a partition of the set received by its parent, and it works on such a subset to further partition it for its children nodes. When a leaf node is reached, the final classification is performed. It is worth noting that firstorder perceptrons split the training set by planes, whereas $n$-order perceptrons use $n$-dimensional surfaces, also known as hyperplanes. In Figure 3, such an architectural scheme is shown.

The AHNT is automatically grown during the learning phase; it is not necessary to choose a priori the number of nodes and their links. The hybrid structure guarantees a minor number of internal nodes and overcomes the classical problems of feedforward neural networks, since it does not require any a priori information about the number of neurons, hidden layers, or neuron connections.

The learning phase consists of feeding the AHNT with vectors defined by all 7 second- and third-order normalized central moments of the blob image (Liao \& Pawlak, 1996), defined as

$$
\eta_{a b}=\frac{\mu_{a b}}{\mu_{00}^{(a+b) / 2+1}} \quad \forall a, b \mid a+b \in\{2,3\},
$$




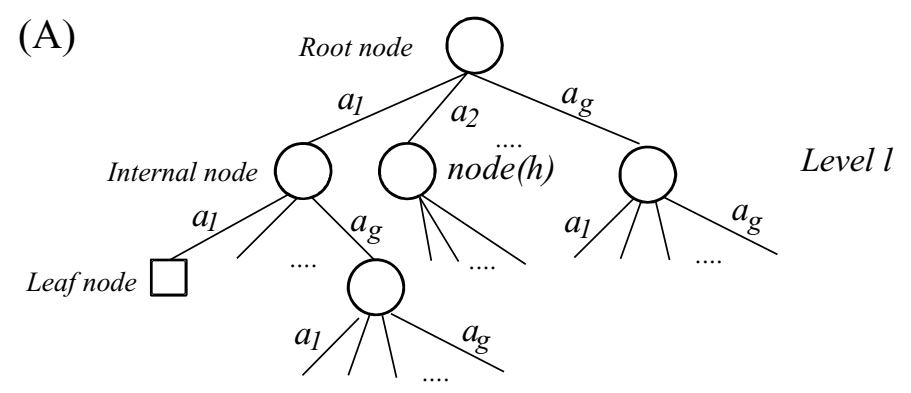

(B)

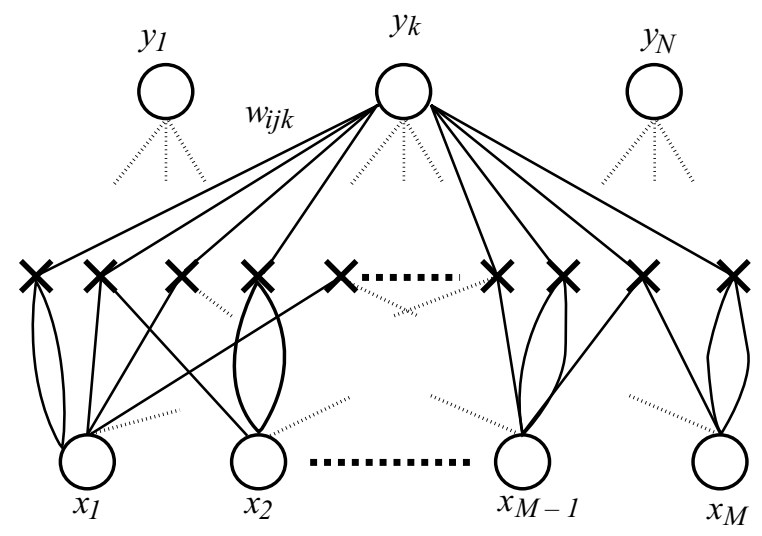

Figure 3. (A) A neural tree and (B) the structure of a single tree node.

where $\mu_{a b}$ are the central moments,

$$
\mu_{a b}=\sum\left(x-x_{c}\right)^{a}\left(y-y_{c}\right)^{b},
$$

and $\left(x_{c}, y_{c}\right)$ are the coordinates of the barycenter of the blob, while the sum cycles over all the $(x, y)$ coordinates of the pixel composing the blob. These features have been proven to describe the most salient characteristics of the blob shape, being invariant across translations and scale transformations.

The object classification obtained on $N$ consecutive frames of the input sequence is analyzed by a winner-takesall procedure. The class that receives the maximum number of occurrences is chosen to identify the detected object. The parameter $N$ is experimentally determined, and it represents a trade-off between the classification robustness and the real-time constraints (current solution uses $N=20$ ).

The obtained classification is used by the behavior understanding module to switch on the correct analyzer as a consequence of the object class.

\section{TRAJECTORY CLUSTERING}

The multicamera tracking approach described in the previous section is used to robustly gather trajectory data. The raw data can then be processed and organized in a more useful way for high-level processing - for example, for anomalous event detection. At higher levels, it is more suitable for working with data describing the general prop- erties of a possibly large group of trajectories, rather than working with single trajectories. For these reasons, we propose a trajectory-clustering algorithm that can group together trajectories with similar features (spatial coverage, object speed, etc.), thus leading to a more concise description of the patterns of activity detected in the scene.

The definition of a clustering algorithm should involve three main points: (1) choosing a way to represent trajectories and clusters, (2) defining a distance measure between trajectories and clusters, and (3) defining how clusters should be updated.

In the proposed method, trajectories are represented simply by a list of coordinates acquired at fixed time intervals and representing the positions of the moving objects projected on the site map. Clusters are represented in a similar way but also include information on the local variance (the width of the cluster at a given point). With this representation, it is easy to define a proper similarity measure in order to check whether a trajectory belongs to a given cluster, on the basis of the distance minima between the trajectory and the cluster elements. It is also a straightforward matter to define a cluster-updating formula, so that a cluster can be dynamically adapted to the matching trajectories. The full process of cluster updating and the similarity measure are described in detail in Piciarelli and Foresti (2005).

Since our final aim is to study trajectories for behavior modeling, we use a data representation that can highlight similarities between activities. In particular, we claim that 


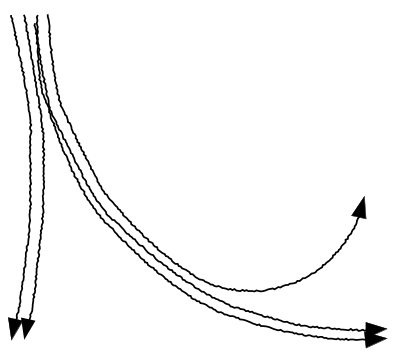

(A)

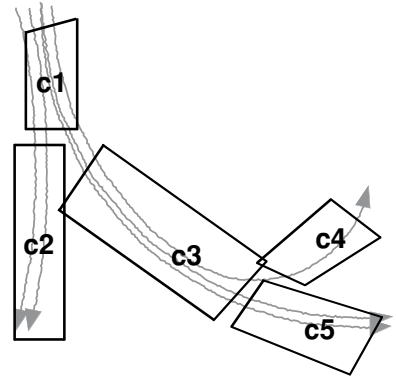

(B)

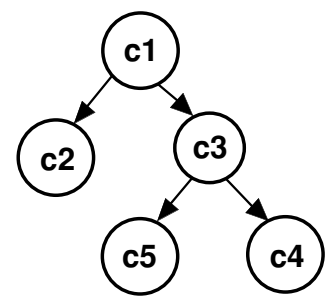

(C)

Figure 4. Representing trajectories as trees of clusters.

in many real-world environments, the clusters are not totally independent of each other, since they often share pieces of trajectories, generally due to environmental constraints (e.g., all the trajectories can share the same origin in the proximity of a gate). A behavior analysis system should be aware of these shared features; in particular, we chose to explicitly model common prefixes (where a prefix is the initial part of a cluster), because the identification of those prefixes will make it possible to make predictions regarding the future movements of an object. We chose to model each common prefix with a cluster, in such a way that trajectories are no longer represented by simple clusters, but by groups of clusters organized in a treelike structure (as shown in Figure 4).

The whole process of building trees of clusters is summarized in Figure 5. When a new trajectory is detected, its similarity to each of the root-node clusters is computed, in order to see whether the trajectory falls inside a cluster. If a match is not found, a new cluster is instantiated; otherwise, the cluster is updated with the incoming data. The system continuously computes the similarity measure in order to check whether the trajectory leaves the cluster or not. If it exits from the cluster's end, a new match is searched for among the children of the just-left cluster (e.g., in Figure 4, if a trajectory exits from cluster c1, a new match is searched for with c2 and c3). Otherwise, if the trajectory exits far from the end of the cluster, the cluster itself is split into two different parts.

\section{BEHAVIOR UNDERSTANDING}

In the previous sections, low-level processing techniques were described for the acquisition of data about moving objects. In particular, objects are classified with a neural network using a predefined taxonomy (e.g., people and vehicles), and their trajectories are grouped into clusters. This data-gathering step is needed to feed the higher level analysis modules, through which we plan to obtain a highlevel, semantic interpretation of the activities in the monitored scene. Since the proposed system has been developed for video surveillance purposes, we are interested mostly in anomalous or explicitly dangerous behaviors. In this case, a behavior is simply a pattern of motion activities, but this kind of information can be successively merged with other behavioral data - for example, facial expression or gesture analysis - obtained by other active vision modules.

The first problem is to choose a suitable way to represent anomalous behaviors. There are two different main approaches: probabilistic definition and explicit definition. The probabilistic approach is based on the consid-

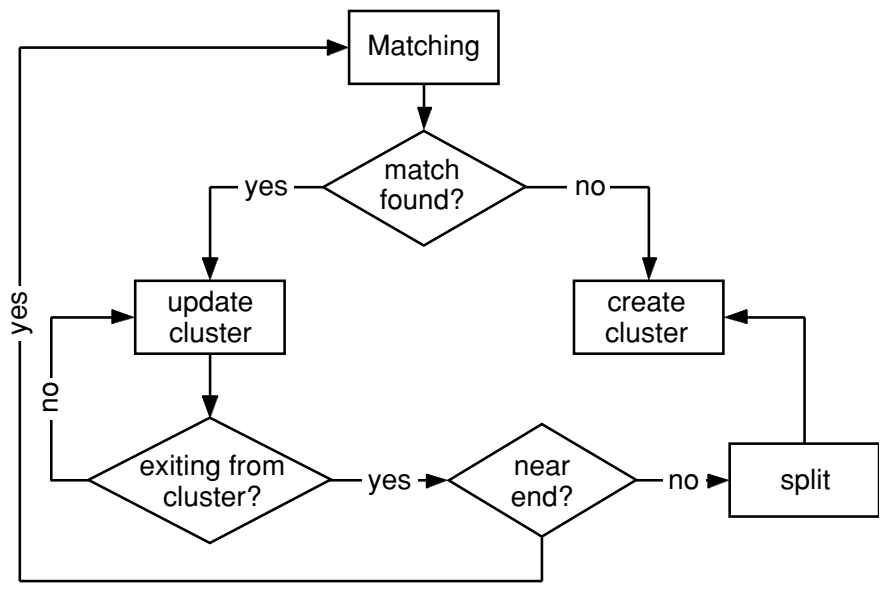

Figure 5. Tree-building process. 
eration that an anomaly is something that by definition happens rarely and differs from the normal patterns of activity. If it were possible to identify the normal patterns, this would straightforwardly lead to the detection of anomalies. The modeling of normal patterns involves a statistical approach, in order to discriminate what is normal from what is not. Of course, not all the anomalous events are dangerous ones, but most probably, a dangerous behavior is an anomalous one, thus justifying the study of anomaly identification in a video surveillance system.

In contrast, the explicit approach is based on the system's having a complete description of all the detectable dangerous events. The events detected in the monitored scene are, thus, matched with those stored in a dangerous events database. This match is performed to check whether such events should be considered as dangerous or not. The main disadvantage of this approach is the need for a priori knowledge of the activities we are looking for, although it is affordable in many specific contexts.

Furthermore, building the database is not a trivial task, even if it does not necessarily need to be performed directly by human operators. For example, complex events, described as relations among many simple events, can be automatically learned by the system, requiring human intervention only to classify them as dangerous or normal activity.

\section{Probabilistic Definition}

As was described above, anomalous behaviors can be detected using a probabilistic approach, in which an anomaly is defined as an event with a low occurrence probability. In this work, we have applied the probabilistic approach to trajectory analysis: The most common trajectories are identified, and an anomalous activity is detected when an object is moving with a trajectory that significantly differs from the built model. We also use information from the object classification module to detect a separate set of most probable trajectories for each kind of object. This is necessary because different types of objects often have very different trajectories; for example, a common trajectory for a man on a sidewalk would be an anomalous trajectory for a car.

The proposed framework for trajectory clustering can easily be used to perform this kind of probabilistic event detection. Recall that clusters of trajectories are organized in a treelike structure. If we keep track of the number of times a cluster is updated, each arc of a tree of clusters can be labeled with a probability defined as

$$
\frac{n_{c}}{\sum_{k} n_{k}}
$$

where $n_{x}$ is the number of times cluster $x$ was matched, $c$ is the child node of the arc, and the index $k$ cycles over all the siblings of the node $c$. This way, we obtain probabilitylabeled trees such as the one shown in Figure 6.

This representation can be used both to make predictions of future movements of an object and to compute the total probability of a trajectory. This probability is defined as the product of the "partial" probabilities that label all the arcs in the path from the root node to the leaf node

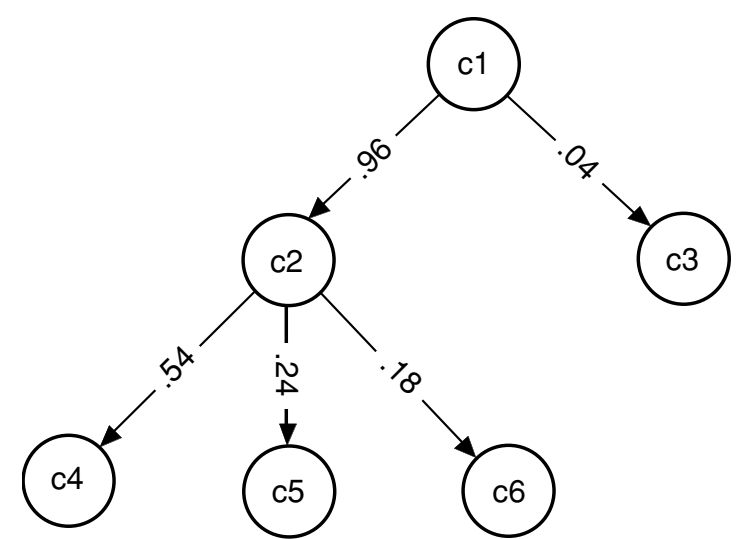

Figure 6. Tree of clusters with probabilities.

associated to the trajectory. For example, in Figure 6, if a trajectory matches cluster $\mathrm{c} 1$, then $\mathrm{c} 2$ and, finally, $\mathrm{c} 5$, its total probability is .96 (probability of going from $\mathrm{c} 1$ to c2) *.24 (probability of going from c2 to c5).

With this approach, anomalous trajectories are defined as those trajectories whose total probability falls below a given threshold.

\section{Explicit Definition}

We now will show an approach based on explicit modeling of dangerous events, which can be used in combination with the probabilistic approach to achieve more robust results.

Two different types of events have been considered: simple events, characterized by the motion (and behavior) of a single object (e.g., vehicles, pedestrians, etc., moving in the monitored environment) and composite events, characterized by interactions among multiple objects. A composite event is, therefore, a complex event generated by a set of temporally and spatially related simple events or an event composed of multiple moving objects (e.g., a group of people, a queue of cars, etc.).

A simple event $E$ is defined over a temporal interval $\left(t_{k}\right.$, $t_{k+n}$ ), and it is composed of a set of features $f_{k}$ belonging to a given object $O_{j}$ observed over a sequence of $n$ consecutive frames as

$$
E\left(t_{k}, t_{k+n}\right)=\left\{f_{k} \in O_{j}\right\}, k \in[1, K], n \in[1, N] .
$$

Examples of real features are the class $C_{i}$ of the detected object, object trajectories, the average object speed $S_{i}$, blob shape descriptors, color histograms, and so forth.

A composite event $C E$ is defined over a wide temporal interval $\left(t_{k}, t_{k+m}\right), m \geq n$, and it is composed of two or more simple events that are spatially and/or temporally correlated:

$$
C E\left(t_{k}, t_{k+m}\right)=\left\{E\left(t_{k+i}, t_{k+j}\right)\right\}, j>i \in[0, m] .
$$

The goal of the proposed system is to detect an event and classify it into a set of tree levels of increasing dangerousness from normal to very dangerous: (1) normal event, 


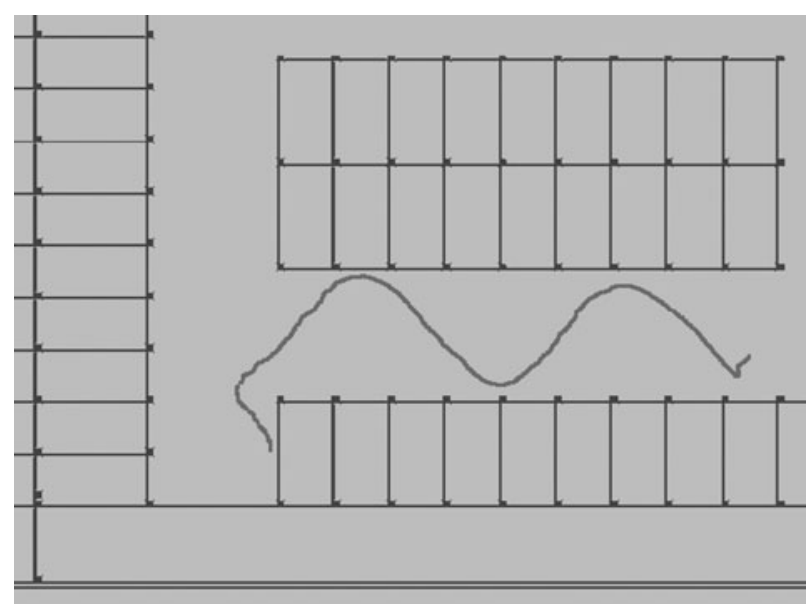

Figure 7. An example of an anomalous trajectory for a pedestrian in a parking lot.

(2) suspicious event, and (3) dangerous event. Suspicious and dangerous events generate two different kinds of alarm signals that are displayed on the man-machine interface (MMI) of a remote operator.

Simple event detection. Let us consider urban environments such as roads, squares, parking lots, and so forth. In these environments, a simple event is normally represented by a vehicle, a bus, a motorcycle, or a pedestrian moving in the monitored area. As a running example, we select a parking lot where normal events are represented by pedestrians walking with typical trajectories (e.g., almost rectilinear for a long number of frames) or vehicles moving and/or stopping in allowed areas. Suspicious events are represented by pedestrians walking with trajectories not always rectilinear, pedestrians stopping in a given zone, pedestrians moving around a vehicle, or vehicles moving in the monitored area for more than a given temporal threshold. Dangerous events are represented by pedestrians or vehicles moving or stopping in areas where this is not allowed or pedestrians moving with atypical trajectories (e.g., moving around several vehicles, as shown in Figure 7).

An offline event database (ED) is built from the models of normal, suspicious, and dangerous simple events. For each event, representing the motion of a given object $O_{j}$, a set of features is extracted and stored in the ED. These features have to be defined a priori, and they depend on the characteristics of the observed scene. In particular, the class $C_{i}$ of the object and the trajectory parameters (direction, speed, curvature, etc.) are used as patterns to train a neural network with three different outputs-for example, normal, suspicious, or dangerous events.

An active event database (AED) is built and updated every time a new simple event has been detected. An aging counter is used to eliminate old events from the AED. Each AED entry is classified using the neural network to determine the suspicious or dangerous simple events. These events are signaled directly to a human operator, whereas the others are further analyzed in order to identify potential anomalous composite events.
Composite events. Composite events are represented by a set of simple events that are spatially and/or temporally correlated. An example of a composite event in the context of a parking lot can be given by a sequence of simple events such as the following: (1) A vehicle enters the parking area, (2) the vehicle moves with a given trajectory, (3) the vehicle stops in a given position, (4) a person exits from the vehicle, (5) the person moves in the parking area, and (6) the person exits the parking area. Composite events are divided into two different classes: (1) normal events and (2) unusual events.

At a given time instant, the AED is inspected to find events having some correlations. First, for each stored event, the initial and final instants, $t_{k}$ and $t_{k+n}$, are analyzed in order to select groups of events with some temporal correlations. A temporal threshold varying from a few seconds to some minutes should be defined according to the application constraints. For example, in a parking lot, it is necessary to define the maximum allowed time that a person normally requires to exit from a car after the car has been stopped. Then, from the set of temporally close events, the initial and final points of the associated trajectories are analyzed in order to find out which events are also spatially close. A spatial threshold varying from one to few meters should be defined according to the application constraints. For example, if some simple events, such as pedestrians walking and cars entering or leaving the parking area, have been detected as temporally close, an automatic procedure searches for spatial correlations among those simple events, in order to find composite events.

When a composite event has been detected, its classification is performed by comparing it with a set of models of normal or unusual composite events previously defined by the system operator.

\section{EXPERIMENTAL RESULTS}

To evaluate the proposed system performances, a parking lot around a university building was selected as a test site.

First, we ran a probabilistic-based approach in order to automatically detect unusual behaviors. Then we ran the solution proposed in Foresti, Micheloni, and Snidaro (2004) on both known and unknown events of interest. The aim of this two-step approach was to compare a probabilisticbased approach with an explicit-definition-based one. Since the behavior assessment depended strongly on the class of the object, an AHNT was trained to classify the objects into three main classes: cars, cycles, and pedestrians. Table 1 shows the results of object classification on a large test set (about $10^{3}$ images). Each row represents an

Table 1 Object Classification Results (in Percentages)

\begin{tabular}{lrcc}
\hline & \multicolumn{3}{c}{ System Classification } \\
\cline { 2 - 4 } Object Class & Car & Cycle & Pedestrian \\
\hline Car & 100 & 0 & 0 \\
Cycle & 1 & 90 & 9 \\
Pedestrian & 4 & 12 & 84 \\
\hline
\end{tabular}




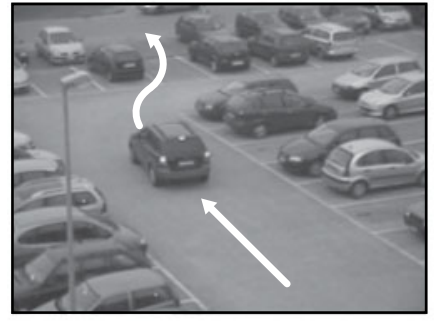

(A)

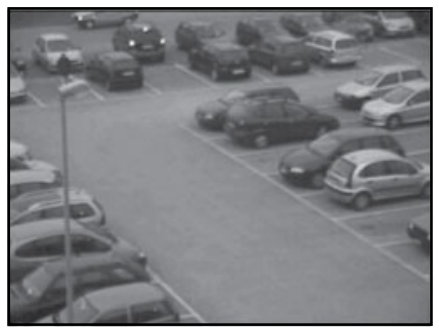

(D)

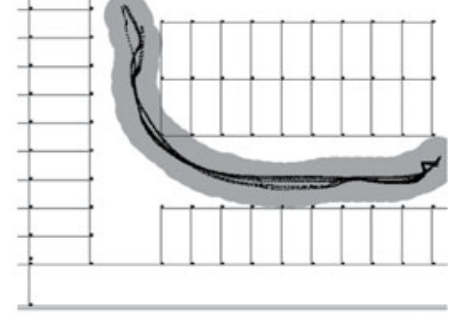

(B)

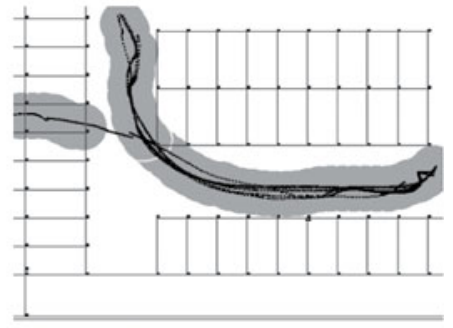

(E)

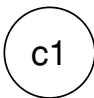

(C)

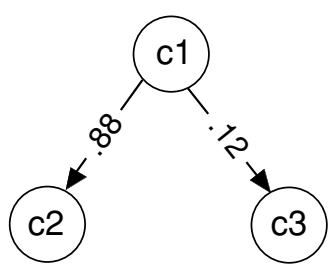

(F)

Figure 8. Assigning probabilities to trajectories.

object class, and the columns show how those objects have been classified by the system.

For the first case, we ran the proposed probabilistic approach for $1 \mathrm{~h}$ in order to acquire a sufficient amount of information (trajectory data) about normal behaviors (Figure 8B). In particular, in this example, only drivethrough trajectories were taken into account. Therefore, the trajectories of parking cars were not considered. On this basis, a tree modeling the typical trajectory clusters was built to detect possible anomalous behaviors. In this case, the tree was composed of a single node, since all the cars performed similar trajectories, leading to the regular exit point (Figure 8C).

Thereafter, we simulated an anomalous behavior by driving a car outside the monitored parking lot through a denied direction. In this situation, the car trajectory initially matched the previously computed model (Figure $8 \mathrm{~A}$ ). Then the car changed its direction, first leading to a parking spot, but soon after exiting through a denied zone (Figure 8D). This event resulted in the modification of the tree of clusters, with the creation of two more nodes (Figure 8F) modeling the trajectories from the forking point to the detected exit zones (Figure 8E). The first node is associated to the previously detected trajectories and has a high probability, whereas the second one represents the newly detected trajectory and has a low probability, since it models only one occurrence. On this basis, it is possible to assert that the new trajectory has a low probability of being detected and, thus, should be considered an anomalous behavior. Concerning the second approach, based on explicit behavior modeling, the system was tested on sequences taken in a parking lot and showing different possible cases. Each sequence was manually la- beled with ground truth data, identifying which kind of simple behavior (normal, suspicious, or dangerous) was happening in the sequences. A feedforward neural network was trained with a back-propagation technique on about 50 patterns representing normal events, 30 patterns for suspicious events, and 20 for dangerous events, for both vehicles and pedestrians. Performances of the proposed system were measured in terms of correct classification in the event recognition process. Table 2 shows the percentage of correct classifications for different normal, suspicious, and dangerous events.

The normal events were further processed by the composite event detector, in order to correlate spatially and/or temporally close simple events. Thereafter, their classification as normal and anomalous behaviors was performed. The results are shown in Table 3 .

To give an overall evaluation of the performance of the two developed approaches, a comparison of reliability between the probabilistic and the explicit-based methods was conducted for simple events. In such a context, as is shown

Table 2

Simple Event Recognition Results

\begin{tabular}{cccc}
\hline & $\begin{array}{c}\text { Normal } \\
\text { Event }\end{array}$ & $\begin{array}{c}\text { Suspicious } \\
\text { Event }\end{array}$ & $\begin{array}{c}\text { Dangerous } \\
\text { Event }\end{array}$ \\
\hline Percentage & 93 & 88 & 89 \\
\hline
\end{tabular}

Table 3

Composite Event Recognition Results

\begin{tabular}{ccc}
\hline & Normal Event & Anomalous Event \\
\hline Percentage & 75 & 82 \\
\hline
\end{tabular}


Table 4

Overall Performance (in Percentages)

\begin{tabular}{lcccccc}
\hline & \multicolumn{2}{c}{ Probabilistic } & & \multicolumn{2}{c}{ Explicit } \\
\cline { 2 - 3 } \cline { 6 - 7 } Events & Known & Unknown & & Known & Unknown \\
\hline Simple & 84 & 83 & & 91 & 5 \\
Composite & - & - & & 78 & 1 \\
\hline
\end{tabular}

in Table 4, the probabilistic approach was able to detect most of the a priori defined suspicious and dangerous events.

In addition, in the presence of suspicious events that were not defined a priori, the probabilistic approach showed good results by detecting the majority of them, whereas the explicit model performed poorly, as was expected.

From the comparison results, it is worth noting how the use of a probabilistic approach, even for the detection of composite events, would be of great help in modeling those behaviors that cannot be defined a priori.

\section{CONCLUSIONS}

In this article, we have presented a computer-based video surveillance system for behavior analysis. Video sequences are acquired by multiple cameras and are processed in order to extract robust information about moving objects - in particular, their classification (e.g., $\mathrm{man} /$ vehicle) and their trajectory. A trajectory-clustering algorithm is then presented; the clustering of trajectories makes it possible to define a simple way of detecting unusual behaviors on the basis of a probabilistic approach. We also presented a dangerous behavior detector based on explicit modeling of what a dangerous event is. The use of both of the approaches together can lead to a reliable classification of the events recorded in a monitored scene. Future work will focus on the application of the probabilistic approach to the detection of composite anomalous behaviors.

\section{REFERENCES}

Ayers, D., \& ShaH, M. (1998). Monitoring human behavior in an office environment. In Proceedings of the Interpretation of Visual Motion Workshop, IEEE Conference on Computer Vision and Pattern Recognition (pp. 65-72). Los Alamitos, CA: IEEE Computer Society Press.

Brand, M., Oliver, N., \& Pentland, A. (1997). Coupled hidden Markov models for complex action recognition. In Proceedings of the IEEE Conference on Computer Vision and Pattern Recognition (pp. 994-999). Los Alamitos, CA: IEEE Computer Society Press.

BuxTON, H., \& GoNG, S. (1995). Visual surveillance in a dynamic and uncertain world. Artificial Intelligence, 78, 431-459.

ChleQ, N., \& Thonnat, M. (1996). Real-time image sequence interpretation for video-surveillance applications. In Proceedings of the IEEE International Conference on Image Processing (Vol. 3, pp. 801-804). Los Alamitos, CA: IEEE Computer Society Press.

Collins, R. T., Lipton, A. J., Fujiyoshi, H., \& Kanade, T. (2001). A system for video surveillance and monitoring. Proceedings of the IEEE, 89, 1456-1477.

ENSER, P. (2000). Visual image retrieval: Seeking the alliance of conceptbased and content-based paradigms. Journal of Information Science, 26, 199-210.

Foresti, G. L., \& Dolso, T. (2004). An adaptive high-order neural tree for pattern recognition. IEEE Transactions on Systems, Man, \& Cybernetics: Part B, 34, 988-996.

Foresti, G. L., \& Gentili, S. (1999). Noise-robust and invariant object classification by the high-order statistical pattern spectrum. International Journal of Pattern Recognition \& Artificial Intelligence, 13, 1219-1232.

Foresti, G. L., Micheloni, C., \& Snidaro, L. (2004). Event classification for automatic visual-based surveillance of parking lots. In Proceedings of the IEEE International Conference on Pattern Recognition (Vol. 3, pp. 314-317). Los Alamitos, CA: IEEE Computer Society Press.

Foresti, G. L., Micheloni, C., Snidaro, L., Remagnino, P., \& Ellis, T. (2005). Advanced image and video processing in active video-based surveillance systems. IEEE Signal Processing Magazine, 22, 25-37.

GAO, J. B., \& HARRIS, C. J. (2002). Some remarks on Kalman filters for the multisensor fusion. Information Fusion, 3, 191-201.

HerzoG, G. (1992). Utilizing interval-based event representations for incremental high-level scene analysis. In M. Aurnague, A. Borillo, M. Borillo, \& M. Bras (Eds.), Proceedings of the 4th International Workshop on Semantics of Time, Space, and Movement and SpatioTemporal Reasoning (pp. 425-435). Toulouse: Université Paul Sabatier, Groupe Langue, Raisonnement, Calcul.

LiaO, S. X., \& PaWlaK, M. (1996). On image analysis by moments. IEEE Transactions on Pattern Analysis \& Machine Intelligence, 18, 254-266.

MAHONEN, P. (1999). Wireless video surveillance: System concepts. In Proceedings of the IEEE International Conference on Image Analysis and Processing (pp. 1090-1095). Los Alamitos, CA: IEEE Computer Society Press.

Piciarelli, C., \& Foresti, G. L. (2005). Toward event recognition using dynamic trajectory analysis and prediction. In Proceedings of the IEEE International Symposium on Imaging for Crime Detection and Prevention (pp. 131-134). Los Alamitos, CA: IEEE Computer Society Press.

Rosin, P. L. (2002). Thresholding for change detection. Computer Vision \& Image Understanding, 86, 79-95.

(Manuscript received October 3, 2005; revision accepted for publication March 21, 2006.) 